\title{
Nonbacterial Thrombotic Endocarditis Concomitant with Repeated Systemic Embolization That Received Palliative Care Based on the Antemortem Diagnosis
}

\author{
Nao Shibata ${ }^{1}$, Kensuke Matsumoto ${ }^{1}$, Satoru Kitamura ${ }^{2}$, Akihiro Sakashita ${ }^{3}$, \\ Yoshiyuki Kizawa $^{3}$ and Ken-ichi Hirata ${ }^{1}$
}

\begin{abstract}
:
A 67-year-old woman was admitted to our hospital due to an acute onset of consciousness disturbance, aphasia and left hemiplegia. Computed tomography revealed multiple systemic infarctions, including brain, kidney and spleen. Transesophageal echocardiography revealed vegetations attached to the mitral valve leaflets, which was suspected to be the embolic source. Repeated blood cultures were negative, and advanced lung cancer was incidentally revealed by computed tomography. She was then diagnosed with nonbacterial thrombotic endocarditis (NBTE) based on the overall clinical picture. Subsequently, extensive systemic embolization repeatedly occurred, and she eventually died 25 days after admission. The autopsy proved NBTE and advanced-stage lung adenocarcinoma.
\end{abstract}

Key words: nonbacterial thrombotic endocarditis, systemic embolization, lung cancer, palliative care, antemortem diagnosis

(Intern Med 57: 3559-3563, 2018)

(DOI: 10.2169/internalmedicine.1381-18)

\section{Introduction}

Nonbacterial thrombotic endocarditis (NBTE), first described in 1888 by Armand Trousseau, is a rare condition characterized by aseptic masses composed of fibrin and platelets on the heart valves (1). The definitive diagnosis is generally made by the demonstration of platelet thrombi at an autopsy or by surgical specimens pathologically. It has been reported that approximately $80 \%$ of NBTE cases are associated with advanced cancer (2), with autoimmune disease, tuberculosis, uremia, and acquired immune deficiency syndrome (AIDS) also reported to be the associated with NBTE (3).

Although the pathogenesis of NBTE remains unclear, it is postulated that circulating cytokines, such as tumor necrosis factor and interleukin-1, released from underlying malignant diseases or autoimmune processes may cause valvular endothelial damage during a hypercoagulable state, thereby resulting in the deposition of thrombus on the heart valves (4). Compared with infective endocarditis, vegetations in NBTE are more easily dislodged and have a strong tendency to embolize $(4,5)$. However, because infective endocarditis, and NBTE share relatively common clinical features, the antemortem diagnosis of NBTE is extremely difficult.

In this case report, we present a case with an antemortem diagnosis with NBTE associated with advanced lung cancer that successfully received extensive palliative care based on this diagnosis.

\section{Case Report}

A 67-year-old-woman with rheumatoid arthritis was admitted to our hospital due to the acute onset of conscious-

\footnotetext{
${ }^{1}$ Division of Cardiovascular Medicine, Department of Internal Medicine, Kobe University Graduate School of Medicine, Japan, ${ }^{2}$ Department of Diagnostic Pathology, Kobe University Graduate School of Medicine, Japan and ${ }^{3}$ Department of Palliative Medicine, Kobe University Graduate School of Medicine, Japan

Received: April 14, 2018; Accepted: May 13, 2018; Advance Publication by J-STAGE: August 10, 2018

Correspondence to Dr. Kensuke Matsumoto, kenmatsu@med.kobe-u.ac.jp
} 

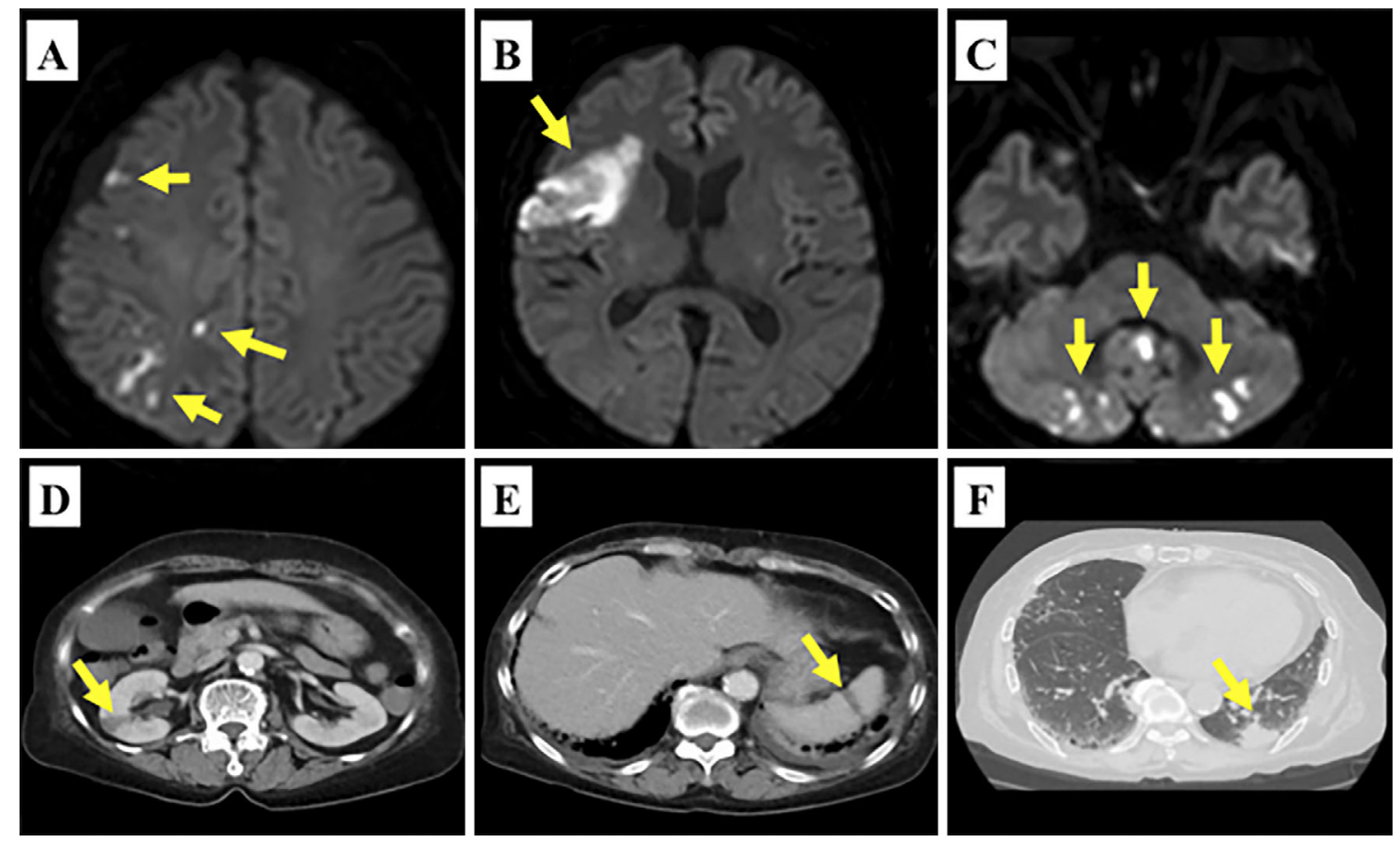

Figure 1. Diffusion-weighted magnetic resonance imaging showed multiple high-intensity signal lesions in the right frontal lobe, temporal lobe and cerebellum (A-C: yellow arrows). Contrast-enhanced computed tomography showed low-density areas in the right kidney (D: yellow arrow) and spleen (E: yellow arrow). Lung tumor in the left inferior lobe was incidentally detected (F: yellow arrow).

ness disturbance, aphasia and left hemiplegia. She had suffered from intermittent fever of $38^{\circ} \mathrm{C}$ and general fatigue for approximately 1 month before the admission. On admission, her blood pressure was $187 / 84 \mathrm{mmHg}$, pulse rate was 94 beats/min, oxygen saturation was $96 \%$, body temperature was $37.1^{\circ} \mathrm{C}$ and Glasgow Coma Scale was E2 V3 M6. On a visual inspection, peripheral vascular obstruction of the fingers, palms, toes and soles were observed, which suggested multiple systemic embolization. On a physical examination, grade II/VI systolic regurgitant murmur was auscultated at the apex. A laboratory examination showed an inflammatory reaction with a white blood cell count of $170 \times 10^{3} / \mu \mathrm{L}(90 \%$ neutrophils) and a C-reactive protein level of $9.41 \mathrm{mg} / \mathrm{dL}$. Thrombotic workup revealed an elevated D-dimer level of $49.2 \mu \mathrm{g} / \mathrm{mL}$.

Magnetic resonance imaging of the brain showed cerebral and cerebellar infarctions of multiple vascular territories (Fig. 1A-C), and contrast-enhanced computed tomography revealed right renal and splenic infarctions (Fig. 1D-E), consistent with multiple cardiogenic embolisms. She underwent transthoracic echocardiography for the assessment of the embolic source, and valvular vegetations on the mitral leaflets were suspected. For a further assessment of the embolic source, transesophageal echocardiography was then performed, which revealed valvular vegetations attached to both the anterior and posterior mitral leaflets with sizes of $6.7 \times$ 7.4 and $11.7 \times 5.2 \mathrm{~mm}$. These vegetations interfered with the coaptation of the mitral leaflets, leading to moderate mitral regurgitation (Fig. 2).

Based on the clinical course of a prolonged intermittent fever, multiple embolization and an inflammatory reaction, an interim diagnosis of infective endocarditis was made, and antibiotics were initially introduced. However, she showed no signs of infection throughout the clinical course, and no organisms were detected from repeated blood cultures.

Computed tomography revealed advanced lung cancer in the left inferior lobe with multiple mediastinal lymph node metastasis (Fig. 1F). Based on the medical background and clinical context, she was diagnosed with NBTE associated with advanced lung cancer. Even after the discontinuation of antibiotic treatment, transthoracic echocardiography showed no progression of mitral regurgitation nor valve destruction throughout the clinical course. Because hemorrhagic cerebral infarction was proved by subsequently performed brain computed tomography, we were unable to introduce anticoagulant therapy. Subsequently, her consciousness status further decreased, and brain magnetic resonance imaging revealed multiple recurrences of strokes. Peripheral embolization of the fingers, palms, toes and soles repeatedly occurred, and her tissue necrosis gradually worsened (Fig. 3). Because she suffered from intractable pain due to repeated multiple peripheral ischemia and necrosis, morphine hydrochloride hydrate was continuously administered to relieve the distal pain. In addition, the palliative care team mentally supported not only the patient but also her family members. Under extensive palliative care, she eventually died 25 days after her admission.

The autopsy revealed the valvular vegetations broadly attached to the both mitral leaflets (Fig. 4) along with lung cancer in the left inferior lobe on a macroscopic examina- 

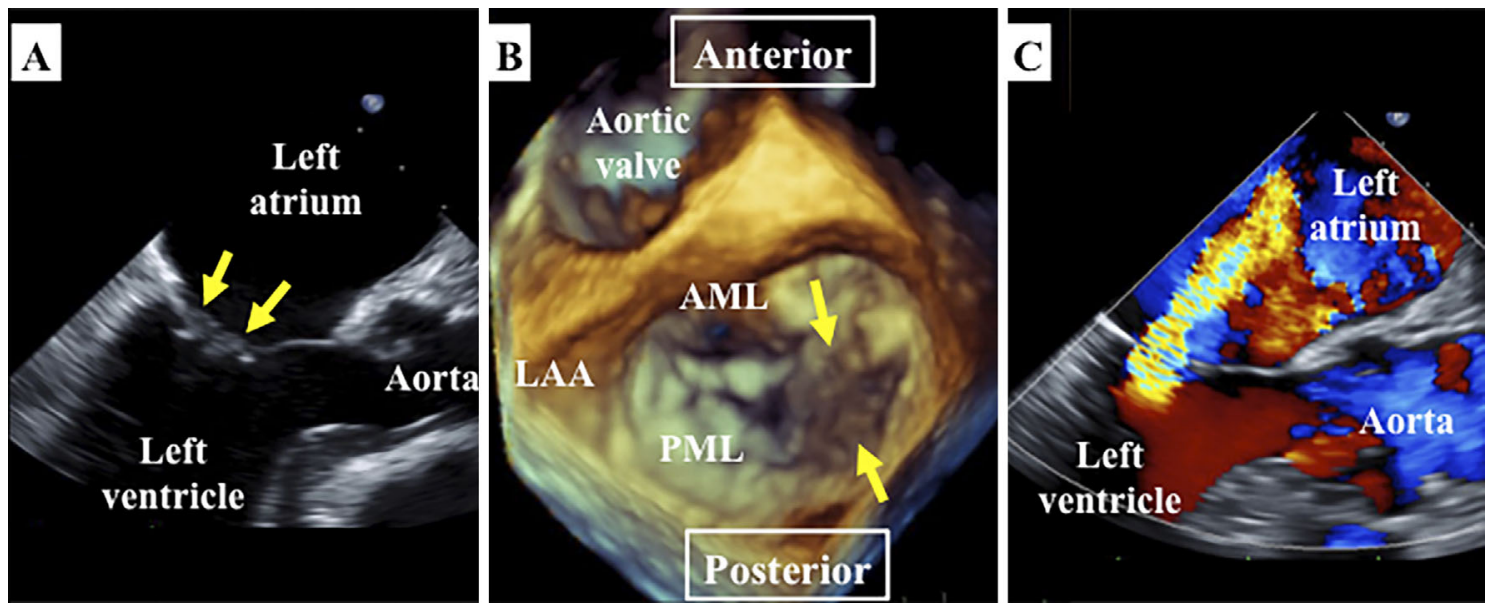

Figure 2. Transesophageal echocardiography showing vegetations attached to the anterior and posterior mitral leaflets (A: mid-esophageal long axis view; the yellow arrows indicate vegetations). Three-dimensional reconstruction imaging from the "surgeon's view" showing vegetations attached to the medial side of both mitral leaflets (B). Color Doppler imaging from the mid-esophageal longaxis view showing moderate mitral regurgitation (C). LA: left atrium, LV: left ventricle, Ao: aorta, AML: anterior mitral leaflet, PML: posterior mitral leaflet, LAA: left atrial appendage
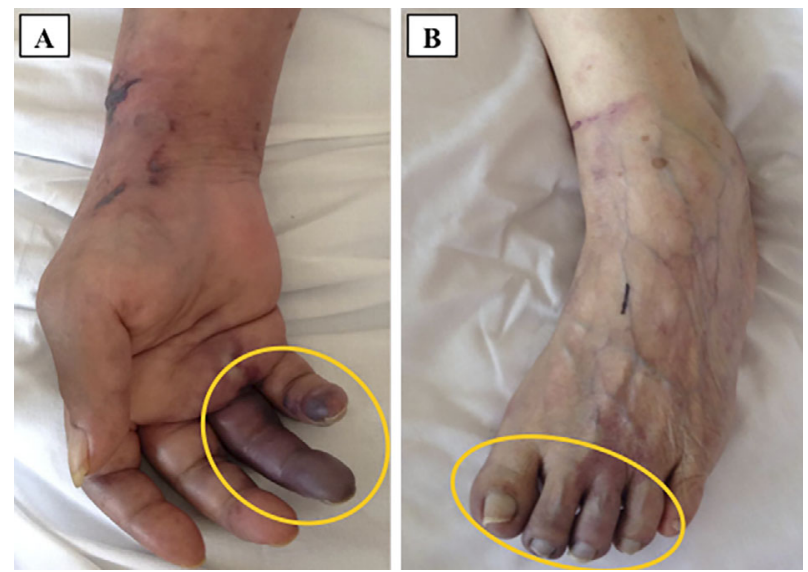

Figure 3. Peripheral embolization on the fingers, palms and toes was observed on admission. A shows the right hand, and $B$ shows the left foot.

tion (Fig. 5). Histologically, the vegetations were composed of fibrin and platelets without bacteria or malignant cells (Fig. 4C). There was no destruction of the valve leaflets, which is quite consistent with the diagnosis of NBTE. Furthermore, the autopsy revealed subacute inferior myocardial infarction consistent with systemic embolization (Fig. 4D and E).

\section{Discussion}

It is sometimes difficult to distinguish NBTE from infective endocarditis, as there are no specific laboratory tests for NBTE, and the presence of a general fever due to the underlying malignancy makes it difficult to clinically suspect NBTE. Although echocardiography is useful for detecting valvular vegetations, it is usually difficult to distinguish a sterile thrombus from infective vegetations. Although we need to rule out blood culture-negative endocarditis, multiple negative blood cultures despite no antibiotics administration aid in the diagnosis of NBTE, as shown in this case. Therefore, when we find blood culture-negative valvular vegetations in a patient with malignancy or auto-immune diseases, we should consider the possibility of NBTE rather than infective endocarditis. Thus, we believe that the barriers to the antemortem diagnosis of NBTE can be overcome through these careful clinical considerations.

Several differences between NBTE and infective endocarditis with regard to the morphological features and clinical presentation have been reported. The vegetations of NBTE are generally small $(<1 \mathrm{~cm}$ in diameter), broad-based, irregular in shape and easily friable, thus frequently leading to embolization. Because there is little inflammatory reaction at the site of the attachment, the vegetations are suspected to be easily dislodged (4). Although systemic embolization can occur in both patients with NBTE and infective endocarditis, embolization in NBTE is known to be more often symptomatic, multiply occurring and widely distributed than infective endocarditis $(1,6,7)$. The common sites of embolization include the spleen, kidney, skin and extremities, which are sometimes painful $(1,8,9)$. In contrast, valve destruction is generally uncommon with NBTE and thus rarely causes significant hemodynamic consequences and heart failure compared with infective endocarditis (5). The autopsy findings of the present case are consistent with those of previous reports. There was no valve destruction, and it was suspected that the broad-based vegetations interfered with the coaptation, potentially causing mitral regurgitation in this case.

One eradicative treatment for NBTE is surgical valve replacement or valve plasty. It is also necessary to control the 


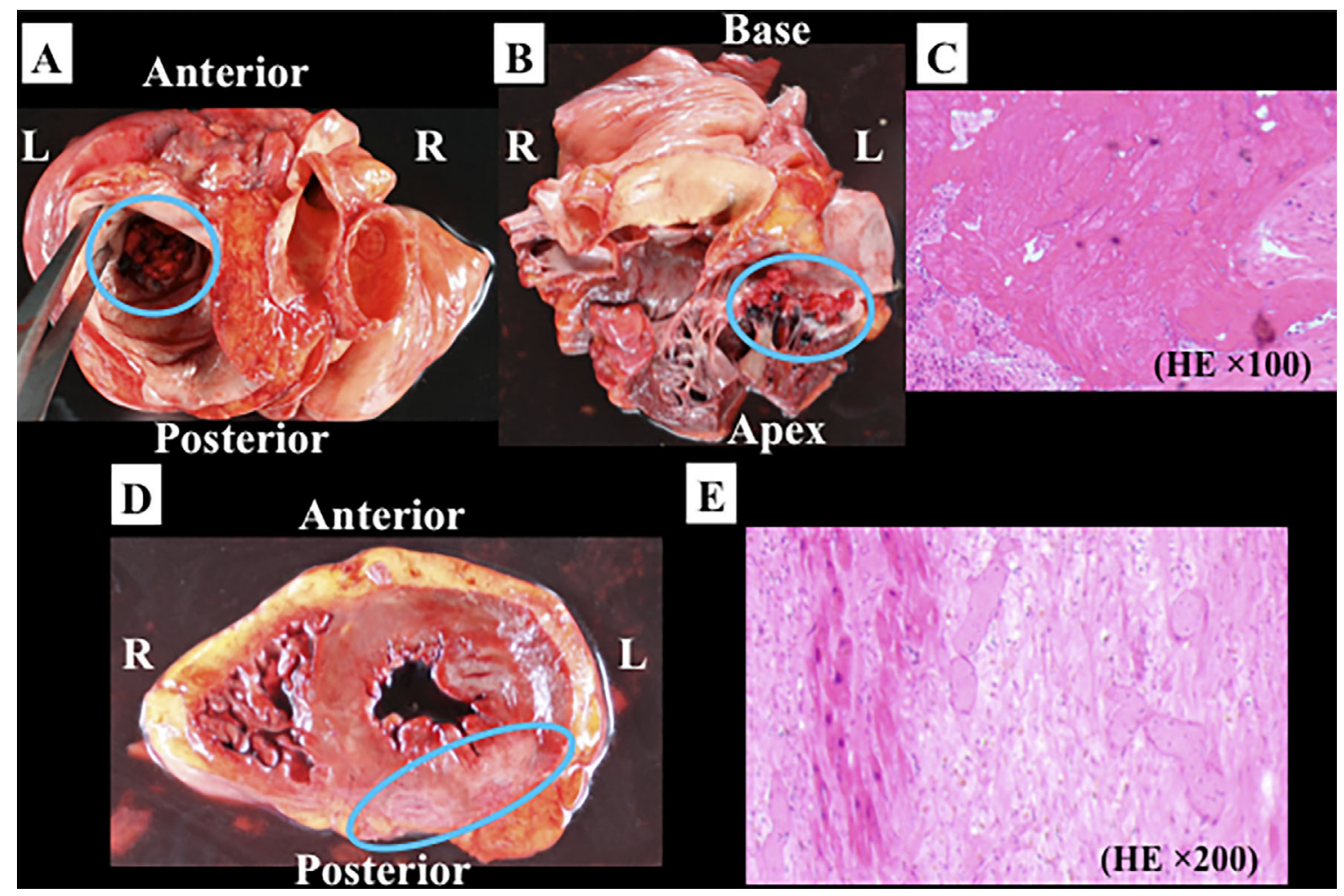

Figure 4. The autopsied heart is shown in this figure. Multiple vegetations were broadly attached to the mitral leaflets (blue circles) (A: horizontal section from the cranial view; B: coronal section). C shows the microscopic examination of the resected vegetation [Hematoxylin and Eosin (H\&E) staining $\times 100]$. Histologically, the vegetations were composed of fibrin and platelets without bacteria or malignant cells. D shows a horizontal section of the heart. Subacute inferior myocardial infarction is indicated with a blue circle. Microscopically, denaturing of myocardial fibers, infiltration of inflammatory cells and fibrosis were detected $(E)$. R: right, L: left, HE: H\&E staining

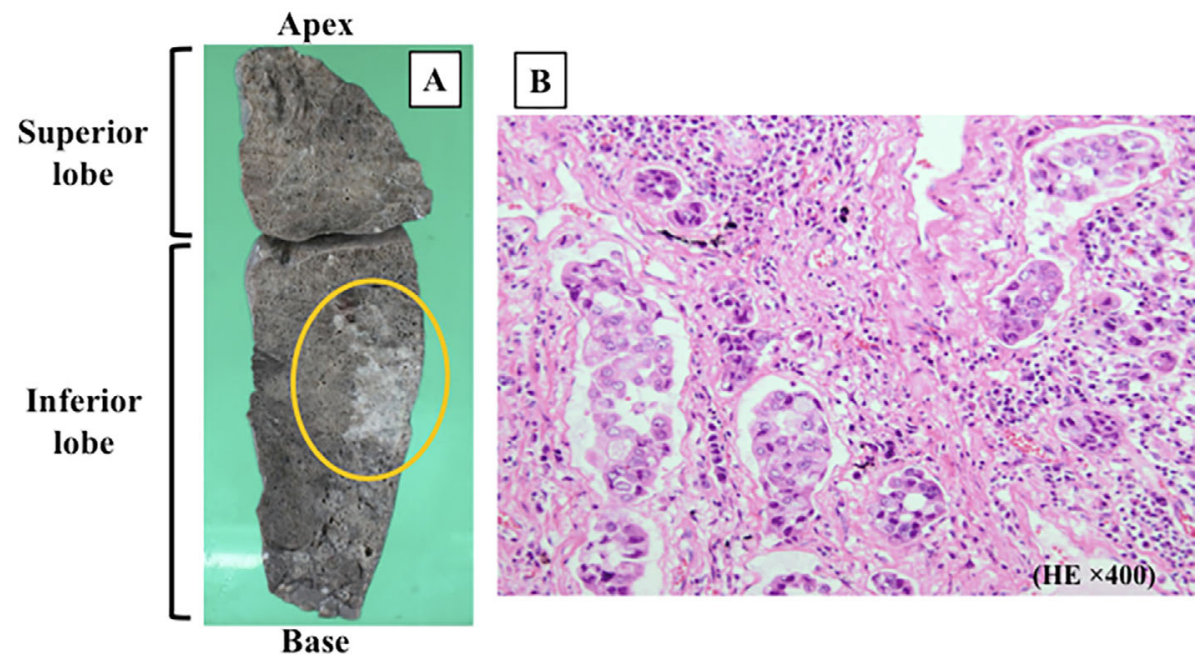

Figure 5. The autopsied left lung is shown in this figure. Macroscopically, the white unclear lesion in the left inferior lobe are consistent with lung cancer (A). Microscopically, atypical cells containing enlarged eccentric nucleus were observed. The growth pattern was lepidic, acinous, nubbly and solitary, which was consistent with invasive lung adenocarcinoma (B: H\&E staining $\times 400$ ).

underlying disease, as NBTE generally recurs otherwise (10). Although the causative tumor should ideally be removed in patients with malignancy (11), it is not often feasible, as patients with NBTE often cannot undergo sur- gery due to a poor general condition and advanced cancer with metastasis when they present with symptoms of embolization.

Anticoagulant therapy is also performed and is known to 
be effective for preventing systemic embolization associated with NBTE (12). However, anticoagulant therapy either cannot be introduced or must be stopped because of the presence of hemorrhagic infraction in some cases. Unfortunately, because our present patient had inoperable advanced lung cancer and presented with hemorrhagic stroke, there was no way to prolong her life. However, based on the antemortem diagnosis of NBTE, we were able to introduce palliative care and relieve the physical and spiritual pain of the patient and her family members. She ultimately passed away peacefully.

\section{Conclusion}

Because it is often associated with advanced cancer, the prognosis of NBTE is generally poor. NBTE can be regarded as the terminal stage in patients with advanced cancer and can cause intolerable pain due to systemic embolization. Therefore, its early recognition and, when necessary, the introduction of vigorous palliative care is desirable.

The authors state that they have no Conflict of Interest (COI).

\section{References}

1. Lopez JA, Ross RS, Fishbein MC, Siegel RJ. Nonbacterial thrombotic endocarditis: a review. Am Heart J 113: 773-784, 1987.

2. Deppisch LM, Fayemi AO. Non-bacterial thrombotic endocarditis: clinicopathologic correlations. Am Heart J 92: 723-729, 1976.

3. Llenas-Garcia J, Guerra-Vales JM, Montes-Moreno S, et al. [Nonbacterial thrombotic endocarditis: clinicopathologic study of a necropsy series]. Rev Esp Cardiol 60: 493-500, 2007 (in Spanish, Abstract in English).

4. el-Shami K, Griffiths E, Streiff M. Nonbacterial thrombotic endo- carditis in cancer patients: pathogenesis, diagnosis, and treatment. Oncologist 12: 518-523, 2007.

5. Asopa S, Patel A, Khan OA, Sharma R, Ohri SK. Non-bacterial thrombotic endocarditis. Eur J Cardiothorac Surg 32: 696-701, 2007.

6. Snygg-Martin U, Gustafsson L, Rosengren L, et al. Cerebrovascular complications in patients with left-sided infective endocarditis are common: a prospective study using magnetic resonance imaging and neurochemical brain damage markers. Clin Infect Dis 47: 23-30, 2008.

7. De Castro S, Magni G, Beni S, et al. Role of transthoracic and transesophageal echocardiography in predicting embolic events in patients with active infective endocarditis involving native cardiac valves. Am J Cardiol 80: 1030-1034, 1997.

8. Doerner J, Boehme P. Presentation of gastric adenocarcinoma with acute arterial occlusive disease, nonbacterial thrombotic endocarditis and pyogenic liver abscess. J Cancer Sci Ther 8: 031-035, 2016.

9. Singhal AB, Topcuoglu MA, Buonanno FS. Acute ischemic stroke patterns in infective and nonbacterial thrombotic endocarditis: a diffusion-weighted magnetic resonance imaging study. Stroke 33: 1267-1273, 2002.

10. Rabinstein AA, Giovanelli C, Romano JG, Koch S, Forteza AM, Ricci M. Surgical treatment of nonbacterial thrombotic endocarditis presenting with stroke. J Neurol 252: 352-355, 2005.

11. Ito S, Yoshitomi H, Park M, et al. Trousseau syndrome with nonbacterial thrombotic endocarditis in a patient with uterine cancer. Intern Med 52: 1353-1358, 2013.

12. Rogers LR, Cho ES, Kempin S, Posner JB. Cerebral infarction from non-bacterial thrombotic endocarditis: clinical and pathological study including the effects of anticoagulation. Am J Med 83: 746-756, 1987.

The Internal Medicine is an Open Access journal distributed under the Creative Commons Attribution-NonCommercial-NoDerivatives 4.0 International License. To view the details of this license, please visit (https://creativecommons.org/licenses/ by-nc-nd/4.0/).

(C) 2018 The Japanese Society of Internal Medicine Intern Med 57: 3559-3563, 2018 